\title{
Visualization of multi-objective decisions for the optimal design of a pressure swing adsorption system
}

\author{
Antanas Žilinskas ${ }^{\mathrm{a}, *}$, Eric S Fraga ${ }^{\mathrm{b}}$, Joakim Beck $^{\mathrm{b}, \mathrm{c}}$, Audrius Varoneckas ${ }^{\mathrm{a}}$ \\ ${ }^{a}$ Institute of Mathematics and Informatics, Vilnius University \\ ${ }^{b}$ Centre for Process Systems Engineering (CPSE), Department of Chemical Engineering, \\ UCL (University College London) \\ ${ }^{c}$ Department of Statistical Science, UCL (University College London)
}

\begin{abstract}
Optimization based process design tools are most useful when combined with the human engineer's insight. Further insight can be gained through the use of these tools by encouraging the exploration of the design space. Visualization is one technique which makes it easier for an engineer to understand the designs identified by an optimization tool. There are many visualization techniques but most are for individual process designs or for understanding the behavior a design space when a single design objective is considered. Most design problems, however, are multi-objective. This paper presents a multi-objective visualization method and applies it to the industrially relevant design of pressure swing adsorption systems.
\end{abstract}

Keywords: optimization, multi-objective, modelling, decision support.

\section{Introduction}

Computer based industrial process design is a challenging task due to a combination of factors, with complex mathematical models and multiple often conflicting objectives being just two of these factors. Multi-objective optimization tools provide a mechanism by which designs can be identified and trade-off curves generated. These allow the engineer to gain insight into the key characteristics of potentially good designs before moving on to more

\footnotetext{
${ }^{*}$ Corresponding author. Tel.:+37052109332, Fax: +37052729209,

Email address: antanas.zilinskas@mii.vu.lt (Antanas Žilinskas)
} 
detailed simulations and pilot plant tests. Insight is gained by analyzing the designs and the trade-off curves and one of the most useful techniques for analysis is visualization. This paper demonstrates the potential of multidimensional visualization for a case study of industrial relevance.

\section{Mathematical model}

Pressure swing adsorption (PSA) is a cyclic adsorption process for gas separation and purification. PSA systems have the potential of achieving a higher productivity for $\mathrm{CO}_{2}$ capture than alternative separation processes [1], such as absorption. However, an efficient and cost-competitive PSA unit is one that achieves high levels of purity and recovery of the product, two competing objectives [2]. Multi-objective optimization methods are therefore required to generate suitable trade-off curves and that allow an engineer to identify those designs which best resolve the conflict in these objectives.

Optimization based design methods require mathematical models of the system. Mathematical models for PSA processes are governed by partial differential algebraic equations (PDAE). The performance of a PSA process is usually based on its behavior at cyclic steady state (CSS). At CSS, the physical conditions at the end of a cycle are identical to those at the beginning of that cycle. To reach CSS from start-up may take hundreds or thousands of cycles [3]. The simulation of a PSA process, therefore, is computationally challenging since the resulting system of PDAEs that needs to be solved is usually large and stiff. Also, hyperbolic PDEs, which often are used, tend to generate solutions suffering sharp fronts in the gas concentration profile, and non-physical oscillations due to shock waves [4]. Because of this, the task to perform PSA simulation can be very time-consuming; a single simulation to CSS can take minutes, hours, or even days. Most optimization approaches thus either use simplified governing equations or limit their search to a reduced design space [3]. More recently, surrogate modeling techniques have been used to address the computational challenge [5].

Given suitable models and appropriate optimization methods, trade-off curves may be generated. The challenge then becomes one of understanding what characteristics of the designs identified are important for the objectives considered, with the aim of enabling an engineer to choose one or more designs for further, more detailed, analysis. Visualisation becomes a key tool in the engineer's repertoire to address this challenge. 

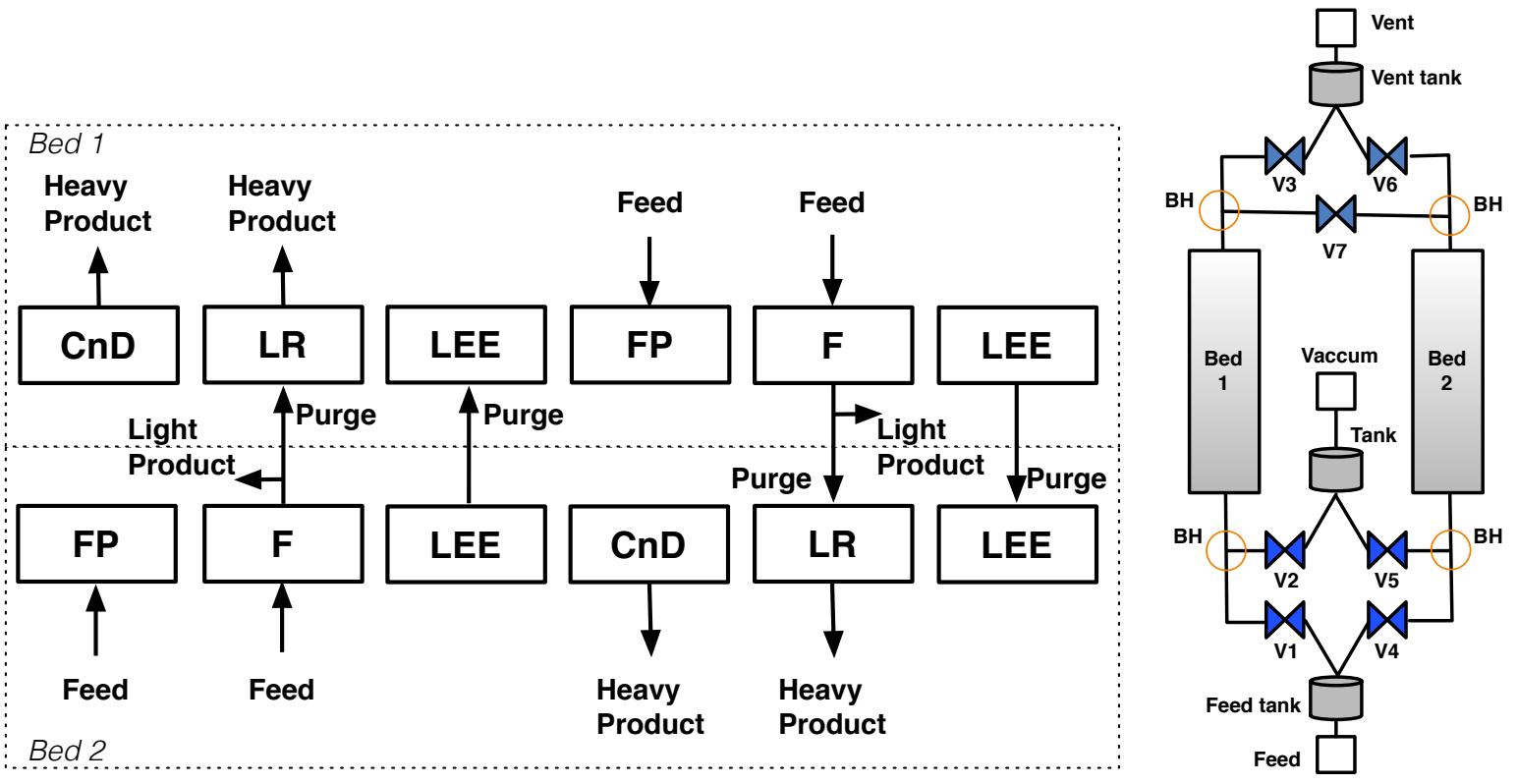

Figure 1: 2-bed/6-step PSA Skarstrom Cycle

To illustrate this challenge and a potential approach, we have chosen a case study from literature that is challenging and sufficiently complex (see [6]) while not intractable computationally. The PSA process is shown in Figure 1 with the process steps shown on the left and the process configuration on the right. This is the PSA Skarstrom cycle with an added pressure equalization step as proposed in [6]. The PSA cycle considered is defined by the following 6 steps: feed pressurization (FP), feed/adsorption (F), light end equalization (LEE), countercurrent depressurization (CnD), light reflux (LR), and ligh end pressurization (LEE). The FP step is characterized by a high-pressure gas mixture entering the bed while not permitting any gas to leave. The $\mathrm{F}$ step is characterized by a high-pressure bed with feed entering the bed. LEE are pressure equalisation steps, and typically used to conserve the energy of the system. $\mathrm{CnD}$ is depressurization with the same flow direction as the adsorption flow. LR is countercurrent low-pressure desorption with light product purge.

The transitions between the process steps are regulated by the stem positions of the valves. The 7 valves involved are shown in the process configuration in Figure 1. The system is symmetrical with the axis of symmetry going 
through the feed and vent units. On both sides of each bed is a bed header which is usually used to ensure a homogeneous flow distribution in the bed. The units labeled "Feed", "Vacuum" and "Vent" provide the boundary conditions for the PSA system. Briefly, the Feed unit is an inlet which provides the gas mixture to separate; the Vacuum unit is an outlet which provides vacuum pressure for the purge and blowdown steps; the Vent unit is an outlet at atmospheric pressure. These three units are referred to as feed units. The tanks next to the feed units are buffering the flow so that the pumps can be operated continuously. The tanks and bed headers are connected by valves which control the flow rates in the system and thus the cycle steps.

This PSA system is considered for the recovery $\mathrm{CO}_{2}$ from the flue gas in a power plant. With the pressure equalization step the $\mathrm{CO}_{2}$ purity can be enriched [6] at the price of a small increase in power consumption. The use of LR steps typically leads to an improved product recovery. One expects that with higher $\mathrm{CO}_{2}$ purity, the system will consume more power at the vacuum pump. The system parameters that are considered fixed are given in Table 2 .

Table 1: System parameters for the PSA unit.

\begin{tabular}{llll} 
Parameter & & value & unit \\
\hline$L_{b}$ & Bed length & 0.12 & $\mathrm{~m}$ \\
$r_{b}$ & Bed radius & 0.0175 & $\mathrm{~m}$ \\
$\epsilon_{b}$ & Bed void fraction & 0.387 & - \\
$V_{C H}$ & Bed header volume & $5.2 \times 10^{-5}$ & $\mathrm{~m}^{3}$ \\
$r_{p}$ & Pellet radius & $9.15 \times 10^{-4}$ & $\mathrm{~m}$ \\
$\epsilon_{p}$ & Pellet void fraction & 0.35 & - \\
\hline
\end{tabular}

The adsorption beds are packed with zeolite $13 \mathrm{x}$ pellets [7]. The stem positions open and close at specific times during the course of a cycle to control the PSA operation. See Table 2 for the stem positions for the different process steps of this 6-step PSA Skarstrom cycle. Here 0 means that the valve is closed, 0.5 half open, and 1 fully open. The PSA cycle is performed through the coordinated operation of the 7 valves. The feed unit supplies a gas mixture of constant pressure, temperature, and feed composition, and therefore held at the initial operating conditions.

The mathematical equations involved describe conservation of mass and energy, pressure profiles, and adsorption kinetics in an adsorbent bed. There 
are mass balance equations for the individual gas components and nonisothermal energy balance equations for the adsorbate in the gas phase, the adsorbate in the solid phase, and for the bed wall. The model is an axial dispersed plug flow model, where the axial dispersion term represents the contribution to axial mixing. The pressure drop along the bed is given by the well-known Ergun equation [3]. The Ergun equation is the steady-state momentum balance of gas flow and relates the pressure drop to the gas velocity along the adsorbent bed. The mass transfer is modeled using the linear driving force (LDF). The LDF model is a linear approximation of the homogeneous diffusion equation for the mass transfer rate. Moreover, the Langmuir adsorption isotherm is used in this study with the data provided in $[8]$.

Table 2: Stem positions for the valves amounting to the different steps. The numbers represent the fraction of the corresponding valve which is open.

\begin{tabular}{llllllll} 
Steps for bed 1 & V1 & V2 & V3 & V4 & V5 & V6 & V7 \\
\hline FP & 1 & 0 & 1 & 0 & 1 & 0 & 0 \\
F & 1 & 0 & $r_{3}$ & 0 & 1 & 0 & $r_{7}$ \\
LEE & 0 & 0 & 0 & 0 & 0 & 0 & 1 \\
CnD & 0 & 1 & 0 & 1 & 0 & 0 & 0 \\
LR & 0 & 1 & 0 & 1 & 0 & $r_{6}$ & $r_{7}$ \\
LEE & 0 & 0 & 0 & 0 & 0 & 0 & 1 \\
\hline
\end{tabular}

The bed header and the tanks are modeled as continuously stirred tanks, and the flow rate in the feed unit is controlled by valve equations:

$$
F=r_{j} c_{v} c_{T} \sqrt{\frac{\left|p_{0}-p_{L_{b}}\right|}{\rho_{f}}} .
$$

Here $r_{j}$ is the stem position, $c_{v}$ the valve coefficient, $p_{0}$ and $p_{L_{b}}$ are the pressures at the two inlets, respectively, $c_{T}$ is the total concentration and $\rho_{f}$ is the fluid density. The pressure in the tank is given by the ideal gas law. The boundary conditions for the gas phase concentrations and the enthalpy are given by the Danckwerts boundary conditions for flow into the bed and the no diffusive flux for flow out of the bed.

For further details on the mathematical model considered in this work, see $[8]$. 
The computational model used relies on the finite volume scheme using 40 volume elements with a Van Leer flux limiter. The simulation time for a single design configuration ranged between 10 minutes to an hour, depending on the design configuration used. The PSA cycles are simulated in succession until CSS has been reached. The backward differentiation formula (BDF) of 5th order is used for time integration.

\section{Statement of the relevant optimization problem}

The PSA design problem is to maximize two conflicting objectives, the product purity and recovery, from $15 \% \mathrm{CO}_{2}-85 \% \mathrm{~N}_{2}$. The $\mathrm{CO}_{2}$ purity and recovery during cycle $k$ is calculated from the total number of moles passing through the vacuum and feed units:

$$
\operatorname{Purity}_{C O_{2}}^{k}=\frac{n_{v a c, C O_{2}}^{k}-n_{v a c, C O_{2}}^{k-1}}{\sum_{i=1}^{N_{c}}\left(n_{v a c, i}^{k}-n_{v a c, i}^{k-1}\right)},
$$

and

$$
\text { Recovery }_{\mathrm{CO}_{2}}^{k}=\frac{n_{v a c, \mathrm{CO}_{2}}^{k}-n_{\text {vac, } \mathrm{CO}_{2}}^{k-1}}{n_{\text {feed }, \mathrm{CO}_{2}}^{k}-n_{\text {feed }, \mathrm{CO}_{2}}^{k-1}},
$$

where $N_{c}$ is the number of gas components, and $n_{v a c, i}$ is the number of moles of component $i$ leaving at the vacuum unit, and $n_{\text {feed }, i}$ the number of moles of component $i$ entering at the feed unit. To calculate the total number of moles you need to integrate the flow rate over time. The two objectives are thus determined by Equation (2) and (3) for the CSS cycle.

The decision parameters for the PSA design problem are given in Table 3.

The described optimal design problem can be mathematically formulated as

$$
\begin{array}{ll}
\mathbf{F}_{\mathbf{P}}=\max _{X \in \mathbf{A}} F(X), & F(X)=\left(f^{1}, f^{2}\right)^{T}, \\
X=\left(x_{1}, \ldots, x_{6}\right)^{T}, & \mathbf{A}=\left\{X: 0 \leq x_{j} \leq 1\right\},
\end{array}
$$

where $x_{j}, j=1,2, \ldots, 6$ denote optimization variables (decision parameters) re-scaled to the unit interval, $F(X)$ is the vector objective function the first component of which $f^{1}(X)$ is $\mathrm{CO}_{2}$ Purity, and the second component $f^{2}(X)$ is $\mathrm{CO}_{2}$ Recovery, and the optimization result $\mathbf{F}_{\mathbf{P}}$ is the Pareto front of the formulated bi-objective optimization problem. For the convenience of the 
Table 3: Decision variables for the PSA design problem.

\begin{tabular}{llll} 
Variable & Description & Range & Unit \\
\hline$r_{7}$ & Purge-to-feed & {$[0,1]$} & - \\
$t_{\text {feed }}$ & Feed/purge time & {$[0,200]$} & $\mathrm{s}$ \\
$F_{\text {feed }}$ & Feed flow rate & {$\left[5 \times 10^{-4}, 8 \times 10^{-3}\right]$} & $\mathrm{mol} \mathrm{s}^{-1}$ \\
$p_{\text {vac }}$ & Vacuum pressure & {$[0.02,0.4]$} & $\mathrm{bar}$ \\
$r_{3}, r_{6}$ & Valve parameter for 3,6 & {$[0.3,1]$} & - \\
$T_{\text {feed }}$ & Feed temperature & {$[290,340]$} & $\mathrm{K}$ \\
\hline
\end{tabular}

readers, who are not experts in multi-objective optimization, we briefly review important facts of the latter; for the comprehensive presentation we refer to $[9,10]$.

The solution of a bi-objective maximization problem, frequently named Pareto front, is the set of Pareto optimal objective vectors, i.e. those vectors both components of which can not be increased. Geometric interpretation of Pareto front is the north-east border of the feasible objective region. The vector of variables corresponding to a Pareto optimal solution is named a Pareto optimal decision.

Solving a bi-objective maximization problem (by means of so called a posteriori methodology [10]) means computing a discrete representation of the Pareto front. From a theoretical point of view, all Pareto optimal solutions are acceptable since none is inferior with respect to any other. However, from an application's point of view, the solutions can frequently be ranked by taking into account factors not included in the mathematical model of the design problem. A representation of the Pareto front found is typically presented to the decision maker who then makes the final decision, i.e selects the most appropriate vector from the set of Pareto optimal decisions.

There are many methods for computing a discrete representation of a Pareto front. To select a suitable one, the available information on the properties of the objective functions should be taken into account. Let us mention two polar cases. In the case of concave smooth objective functions, the methods which generalize the methods of classical mathematical programming are efficient [10]. On the other pole are problems where information about properties of the objective functions is very scarce, and their derivatives are not available. Especially difficult are problems with "noisy" objective functions 
[11], the values of which are e.g. computed by Monte Carlo methods. In such so called black-box optimization case, methods based on statistical models of objective functions seem most appropriate [12].

The applied problem considered in the present paper should not be attributed to these polar classes of problems, however. Since the analytical representation of objective functions is not available their concavity cannot be examined. The application of classical methods is also hardly possible because of not availability of derivatives of the objective functions; their numerical estimation would take too much of computing time because the algorithms of objective functions are themselves computationally intensive. On the other hand, the objective functions in question do not correspond to the black-box model since some regularity can be expected because of underlying physical model. The errors in computation of the objective function values are not so significant that the objective functions to be considered "noisy". A similar situation is typical for applications with mathematical models of high mathematical complexity. Numerous publications recommend in such a cases application of genetic algorithms; we refer to comprehensive presentations in a number of monographs $[9,13,14]$.

NSGA-II (The Non-dominated Sorting Genetic Algorithm-II), proposed by Deb et al. [15], has been selected as the multi-objective GA in this study. It uses a parameter free crowded-comparison operator and elitism to attain diversity. The elitist mechanism combines the best parents with the best offspring. An initial population is created, typically at random. A population is ranked according to non-domination: first, all non-dominated individuals are collected into a single category, in which all individuals are assigned the same fitness value, equal to the category number ( 1 is the best, 2 second best, and so on). This group of individuals in category 1 is put aside, and the procedure is repeated for the non-dominated individuals of the remaining part of the population, for category 2 , and so on. This leads to several layers of non-dominated individuals, called fronts, with the first front being the Pareto front.

The fitness of an individual is thus determined primarily by the category number it belongs to, and secondarily by its ranking within the group of individuals in the category. The ranking within the group is achieved by sorting the individuals with the crowded-comparison operator. This sorting mechanism promotes diversity and achieves well-spread fronts. In the next step of the algorithm, offspring are generated using crossover and mutation operators. This algorithm uses the real-coded genetic algorithm with simulated 
binary crossover and polynomial mutation (see [16]), which requires a few parameters to be specified: crossover rate, crossover index, mutation rate, and mutation index. The new population for the next generation is selected according to the non-dominated sorting (described above). $\mathrm{N}$ offspring are generated and combined with the population of parents and offspring. Then, the $\mathrm{N}$ best individuals are selected from this combined population of size $2 \mathrm{~N}$. Here $\mathrm{N}$ is the population size of the initial population.

The crowded-comparison operator, given in [15], when ranking individuals in group is based on distances in the objective space between all the individuals in the group and two additional boundary points (to improve the spread of the fronts). An individual is considered better than another if the individual has a better non-dominated rank (category number) or if it has a higher crowded-comparison value at the same non-dominated rank.

Because the bi-objective function in this case study is computationally expensive, a small population size, 16, is used. Furthermore, we have used crossover rate $9 / 10$, crossover index 5 , mutation rate $1 / 6$, and mutation index 20. These are the default values given in [15], with one exception: we used a lower crossover index as it produces a higher crossover productivity, i.e., a larger spread of offspring solutions to that of the parents. This may prevent future populations from becoming too homogeneous. These settings are chosen based on our experience with NSGA-II in the study of surrogate modeling [8] for pressure swing adsorption design problems.

\section{Visualization of the optimization results}

The application of the chosen NSGA-II multi-objective algorithm to the problem considered resulted in computing of $N=1584$ two dimensional vectors of objectives $F_{i}$ at the points $X_{i}, i=1, \ldots, N$, the components of which belong to the unit interval: $0 \leq x_{j i} \leq 1$ where the index $j,(1 \leq j \leq 6)$, denotes the number of component of the $i$-th vector.

The subset of $F_{i}, i=1, \ldots, N$ constituted of non-dominated points represents the Pareto front of the considered problem $\mathbf{F}_{\mathbf{P}}$; it consists of $N_{P}=179$ two-dimensional vectors; the corresponding subset of $X_{i}, i=1, \ldots, N$ is denoted by $\mathbf{X}_{\mathbf{P}}$. A graphical drawing of the Pareto front is the standard presentation of results of a bi-objective optimisation problem $[9,10]$. By a visual analysis of the drawing a decision maker can chose an appropriate trade-off between the objectives. Since the Pareto front is represented by a finite number of points, the representation precision crucially depends on the 
number and distribution of points. The drawing of the Pareto front of the considered problem is presented in Figure 2(a). The points are distributed rather densely and uniformly over all Pareto front but has some discontinuity at the beginning of the upper part of the graph which indicates some neighborhood of minimum of $f^{2}(\cdot)$. The very precise computation of the ends of this discontinuity does not seem important since the most interesting part of the Pareto front is that including the kink. For a better identification of this part of the Pareto front a graph of $f^{1}\left(X_{i}\right)$ and $f^{2}\left(X_{i}\right), X_{i} \in \mathbf{X}_{\mathbf{P}}$ is presented in Figure 2(b) where the horizontal axis is for the indices reordered according to the increase of $f^{1}\left(X_{i}\right), X_{i} \in \mathbf{X}_{\mathbf{P}}$. The kink of the Pareto front is formed by the solutions of the indices of which belong to the interval $(80,140)$.
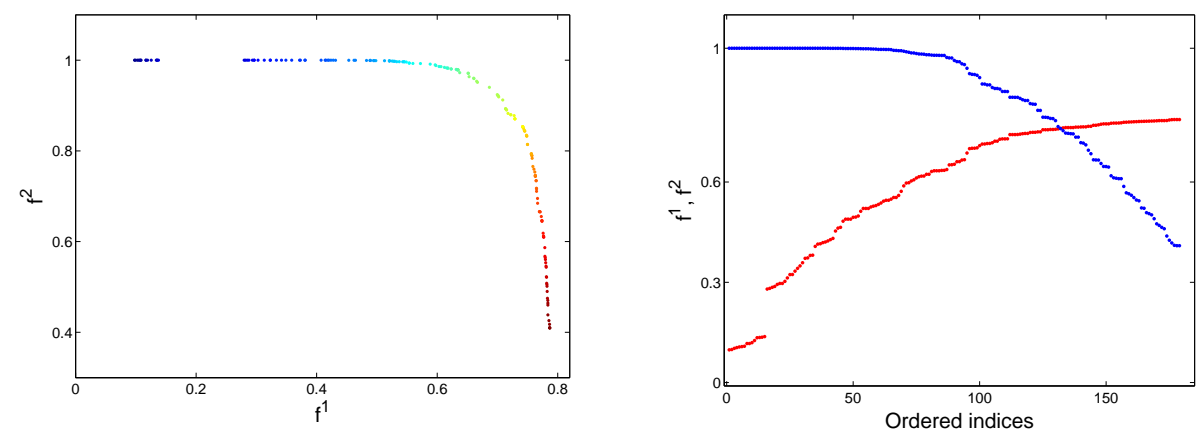

Figure 2: a) Pareto front of the considered problem; b) the graphs of $f^{1}\left(X_{i}\right)$ and $f^{2}\left(X_{i}\right)$, $X_{i} \in \mathbf{X}_{\mathbf{P}}$ with respect to the reordered indices.

By a visual analysis of graphs in Figure 2 an appropriate Pareto solution can be selected as well as the decision $X \in \mathbf{X}_{\mathbf{P}}$ which corresponds to the selected Pareto solution. However, such a choice is not always satisfactory since it does not pay respect to such properties of the corresponding decision as e.g. the location of the selected decision vector in the feasible region $\mathbf{A}$. The analysis of the location of the set of efficient points in A can be especially valuable in cases of structural properties of the considered set important for the decision making. For example, some subsets of A might not be forbidden but may be unfavorable, and that property may not be easy to introduce into mathematical model. The analysis of the properties of the set of efficient points can enable the discovery of latent variables, a relation between which essentially defines the Pareto front.

Numerous statistical methods are developed for the estimation of the 
impact of particular variables to the response in question [17]. Since the considered optimization problem is bi-objective, the Pareto front and the set of the non-dominated decisions are single dimensional manifolds; in other words it is a curve in the six dimensional unit cube. In principle, the parametric description of such a curve could be derived using a least-squares technique. However, the numerical solution of general non-linear least-squares problems is difficult [18]. Therefore an exploratory analysis of the available data seems reasonable here since it may highlight important specific properties of the problem in question.

A suitable method for the discovery of a structure in multidimensional data is visualization $[19,20]$. For the applications of visualization methods in applied optimization, in a context close to the present paper, we refer to $[21,22,23]$. A recent paper [24] discusses broader perspectives of the visualization role in chemometrics.

To get an idea on the location of $\mathbf{X}_{\mathbf{P}}$ in the six-dimensional unit cube, a multidimensional scaling based algorithm [25] has been applied to the twodimensional visualization of a set of six-dimensional points consisting of $\mathbf{X}_{\mathbf{P}}$ and the cube vertices. The multidimensional scaling based visualization algorithm has been applied because it is suitable for highlighting the structure of data sets with up to 10 variables and 1000 points. For theory of multidimensional scaling we refer to $[26,27]$ and for the general advantages of multidimensional scaling based visualization algorithms we refer to [28]. The favorable properties of the multidimensional scaling based visualization are demonstrated also in [29] where images of hyper-cubes and hyper-simplices of various dimensions are presented.

In Figure 3 the two-dimensional image of the vertices of six-dimensional cube is presented which is obtained using a version of the algorithm of multidimensional scaling proposed in [25]. Let us note that the symmetries in the original data are preserved in the image. Our previous experience, e.g. from the investigation presented in [21], suggests that this visualization method reasonably highlights the location of a set of points of interest with respect to the vertices of the hyper-cube.

In the problem considered, the number of points in the set to be visualized was equal to 243; there were 179 points representing the Pareto front and 64 vertices of the hypercube A. The results are presented in Figure 4 where the images of the Pareto points are represented by colored circles (o) and the cube vertices are represented by stars $(*)$.

As it is clearly seen in Figure 4 the set $\mathbf{X}_{\mathbf{P}}$ consists of two subsets. The 


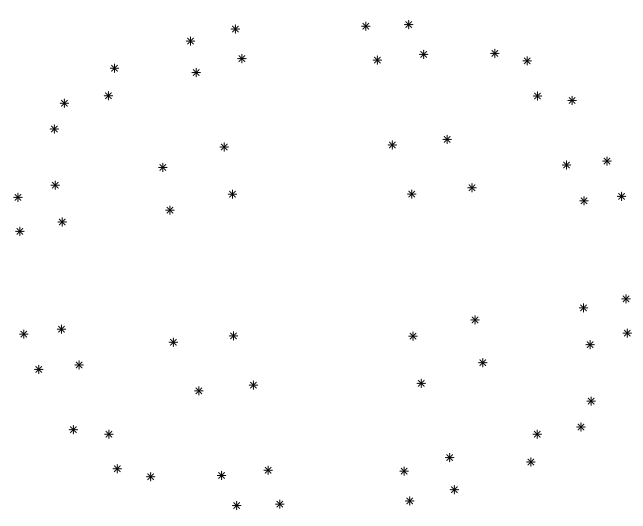

Figure 3: Two-dimensional image of vertices of the six-dimensional hypercube.

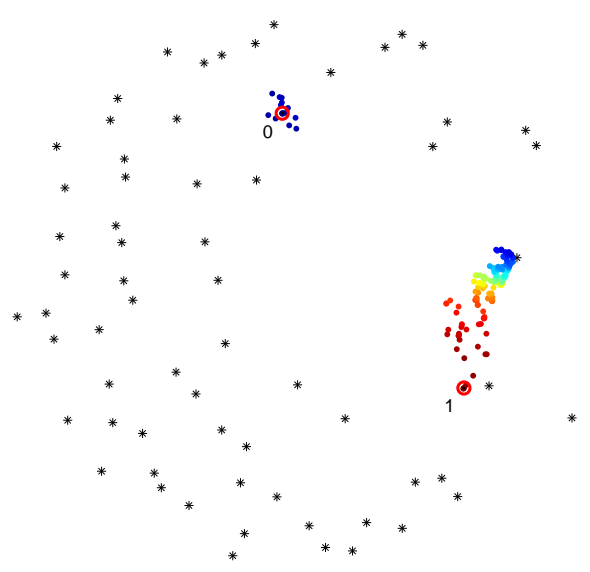

Figure 4: Two-dimensional image of the discrete representation of the set of Pareto optimal decisions, and vertices of the six-dimensional hypercube; the markers 0 and 1 shows the location of minimizers of $f^{2}(\cdot)$ and $f^{1}(\cdot)$ respectively. 
smaller subset is in some vicinity of the minimizer of the second objective function $f^{2}(\cdot)$. The second, larger, subset is located closely to the line segment connecting the points marked by numbers 0 and 1 . The other cube vertices are considerably further from that subset of $\mathbf{X}_{\mathbf{P}}$ than the points marked by 0 and 1 . The latter points represent the vertices of the hypercube $(0,0,1,0,0,1)^{T}$ and $(0,1,1,0,0,1)^{T}$. From the graph it can be guessed that the most significant variable for the selection of a Pareto decision is $x_{2}$, and the values of other variables can be chosen close to the boundary values $x_{1}=x_{4}=x_{5}=0$, and $x_{3}=x_{6}=1$.

\section{The analysis of exploratory guess}

The visualization applied to the available data gives a reason to guess the variable $x_{2}$ as the most significant variable defining a point on the Pareto front. However, visual exploratory approach does not provide reliable conclusions, and a further analysis is necessary either to prove or to reject this guess.

If the guess above is correct, a clearly expressed dependency between the value of $x_{2}$ and the position of the corresponding point on the Pareto front should exist. To indicate such a dependency in Figure 5(a), the values of $x_{2}$ are shown depending on the index of a Pareto optimal solution where the latter are sorted according to the increase of $f^{1}\left(X_{i}\right)$.
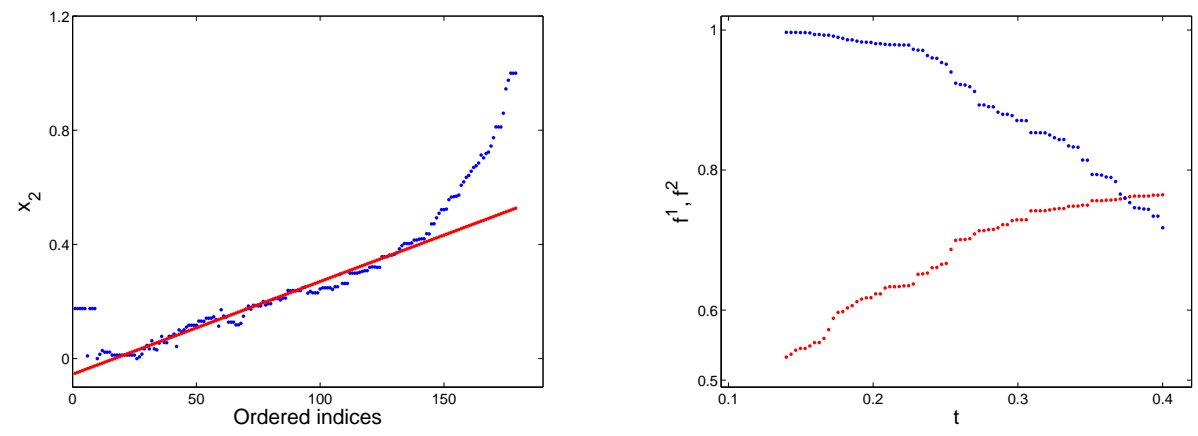

Figure 5: a) The dependency of $x_{2}$ on indices of ordered points in the Pareto front; b) the graphs of $f^{1}(X(t))$ and $f^{2}(X(t))$, where values of $t$ correspond to the kink of the Pareto front.

The linear dependency between $x_{2}$ and the index of a Pareto optimal decision is clearly seen in the Figure 5(a) for the indices which belong to the 
interval $(20,170)$, which is much longer than the interval of interest indicated above. Since the points of the discrete representation are distributed over the Pareto front quite densely and uniformly, all characteristics of interest can be presented as functions of the index as an independent variable. However such a parametric description of problem data has a disadvantage: the independent variable has no interpretation in the engineering terms of problem formulation.

A variable $t$ varying along the line in Figure 5(a) seems well suitable for usage as an independent variable for parametric description of the data of interest. The value of $t$ can be interpreted as a value of $x_{2}$ smoothed along the Pareto front. The values of $t$ in the interval $0.15 \leq t \leq 0.4$ correspond to the kink of the Pareto front. The relationship between $f^{1}\left(X_{i}\right), f^{2}\left(X_{i}\right)$ and corresponding value of $t$ is presented by Figure 5(b). The graphs of $x_{2}(t)$, and $x_{4}(t) 0.15 \leq t \leq 0.4$ are presented in Figure $6(\mathrm{a})$.
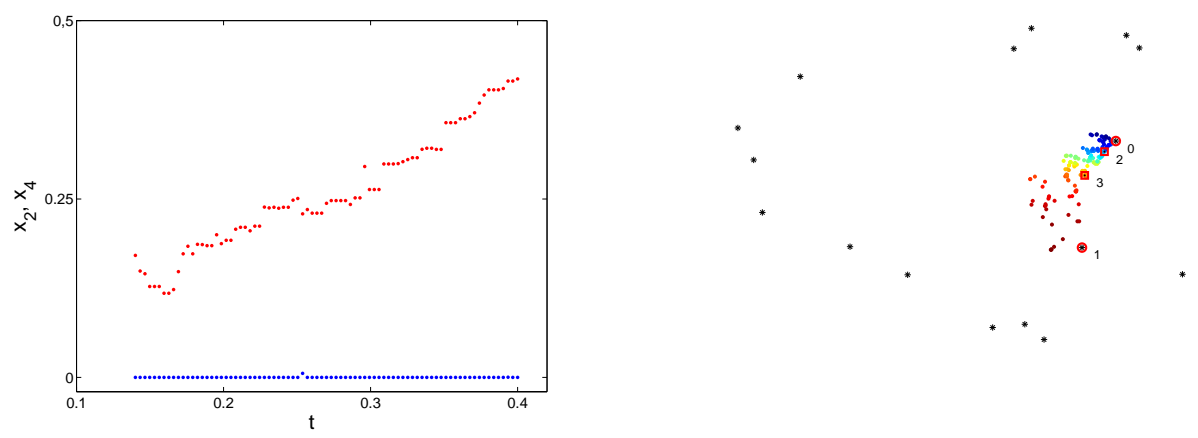

Figure 6: a) The dependency of $x_{2}$ (increasing curve) and of $x_{4}$ (lower graph) on $t$, $0.15 \leq t \leq 0.4$; b) two-dimensional image of the discrete representation of a part of set of Pareto optimal decisions, and vertices of the six-dimensional hypercube where the markers 0 and 1 shows location of closest vertices, and the markers 2 and 3 indicate the decisions of the ends of most interesting part of the Pareto front.

To highlight the location of the most interesting part of the Pareto optimal decisions corresponding to the kink of the Pareto front, the part of image of Figure 3 is presented in Figure 6(b). The images of the closest vertices are marked by 0 and 1 . The points 2 and 3 mark images of the decisions corresponding to the values of $t$ equal to 0.15 and 0.45 correspondingly.

Before the analysis presented above, we expected that a high-temperature adsorbent bed would be advantageous to achieve a high $\mathrm{CO}_{2}$ content in the product stream [30]. Also, we expected that a purge-to-feed would reduce the 
$\mathrm{CO}_{2}$ content of the heavy product. We also knew that the amount of feed gas that enters the system is linear with respect to the feed/purge time, $x_{2}$, which explains why $x_{2}$ displayed such a substantial impact on the performance. For example, long cycle times typically diminish the recovery but lead to a higher purity of the heavy product as the adsorbent beds approach the saturation limit. The feed flow rate, $x_{3}$, which also affects the amount of feed gas that enters, is not as influential, but partially this is because the design constraint given for the flow rate in the design problem. This can be observed in Figure 6(a).

It is however quite surprising in this case that when comparing the optimal design with respect to the purity, against the recovery, on the most interesting segment of the Pareto curve, they only disagree on the feed/purge time, whereas for the other design parameters they concur. Hence, the visualization method revealed that the purity and recovery is only weakly conflicting; in this case the two objectives only contested each other on the feed/purge time.

\section{Conclusions}

This is the first parametrization of the set of Pareto optimal decisions targeted at the optimal design of pressure swing adsorption systems, which is a relevant and challenging design problem. This multi-objective visualization method can be a valuable tool to explore the relationship between competing objectives for the promising designs identified by a bi-objective optimization method. It may enable a decision maker to better understand the relation between different objectives and also their place in the overall design space. This understanding will lead to better informed decisions.

\section{Acknowledgments}

Postdoctoral fellowship of A. Varoneckas is being funded by European Union Structural Funds project Postdoctoral Fellowship Implementation in Lithuania.

\section{References}

[1] D. M. Ruthven, Principles of Adsorption and Adsorption Processes, Wiley-Interscience, 1984. 
[2] G. Fiandaca, E. S. Fraga, S. Brandani, A multi-objective genetic algorithm for the design of pressure swing adsorption, Engineering Optimization, 41(9) (2009) 833-854.

[3] L. T. Biegler, L. Jiang, G. V. Fox, Recent advances in simulation and optimal design of pressure swing adsorption systems, Separation \& Purification Reviews, 33(1) (2005) 1-39.

[4] R. J. LeVeque, Finite-Volume Methods for Hyperbolic Problems, Cambridge University Press, 2002.

[5] J. Beck, D. Friedrich, S. Brandani, S. Guillas, E. S. Fraga, Surrogate based Optimisation for Design of Pressure Swing Adsorption Systems, Proceedings of the 22nd European Symposium on Computer Aided Process Engineering (2012) 1217-1221.

[6] J-H. Park, H-T. Beum, J-N. Kim, S-H. Cho, Numerical analysis on the power consumption of the PSA process for recovering CO2 from flue gas, Industrial \& Engineering Chemistry Research, 41(16) (2002) 4122-4131.

[7] D. Ko, R. Siriwardane, L. T. Biegler, Optimization of a Pressure-Swing Adsorption Process Using Zeolite $13 \mathrm{X}$ for $\mathrm{CO}_{2}$ Sequestration, Industrial \& Engineering Chemistry Research, 42(2) (2003) 339-348.

[8] J. Beck, D. Friedrich, S. Brandani, E. S. Fraga, Multi-Objective Optimisation using Surrogate Models for the Design of PSA Systems, In preparation.

[9] K. Deb, Multi-objective optimization using evolutionary algorithms, Wiley, 2009.

[10] K.M. Miettinen, Nonlinear multiobjective optimization, Kluwer, Dordrecht, 1999.

[11] J. Calvin, A. Žilinskas, One dimensional global optimization for observations with noise, Computers and Mathematics with Applications 50 (2005) 157-169.

[12] A. Žilinskas, A statistical model-based algorithm for black-box multiobjective optimization, International Journal of System Science, 45(1) (2014) $82-93$. 
[13] M. Gen, R. Cheng, Genetic algorithms and engineering optimization, Wiley, 2000.

[14] M. Sakawa, Genetic algorithms and fuzzy multiobjective optimization, Kluwer, 2001.

[15] K. Deb, A. Pratap, S. Agarwal, T. A. M. T. Meyarivan, A fast and elitist multiobjective genetic algorithm: NSGA-II, IEEE Evolutionary Computation, 6(2) (2002) 182-197.

[16] K. Deb, M. Goyal, A combined genetic adaptive search (GeneAS) for engineering design, Computer Science and Informatics, 26(4) (1996) 3045 .

[17] J. Miller, J. Miller, Statistics and Chemometrics for Analytical Chemistry (6th Edition), Pearson Education Canada, 2010.

[18] A. Žilinskas, J. Žilinskas, Interval arithmetic based optimization in nonlinear regression, Informatica 21(1) (2010) 149 - 158.

[19] Ch. Chen, Mapping Scientific Frontiers: The Quest for Knowledge Visualization, Springer, 2014.

[20] G. Dzemyda, O. Kurasova, J. Žilinskas, Multidimensional Data Visualization, Springer, 2012.

[21] A. Žilinskas, E. S. Fraga, A. Mackute, Data analysis and visualisation for robust multi-criteria process optimisation, Computers \& Chemical Engineering 30(6-7) (2006) 1061-1071.

[22] A. Törn, A. Žilinskas, Global optimization, Lecture Notes in Computer Science, 350 (1989) 1-255.

[23] A. A. Zhigljavsky, A. Žilinskas Stochastic global optimization, Springer, N.Y., 2008.

[24] J. Camacho, Visualizing big data with compressed score plots: approach and research challenges, Chemometrics and Intelligent Laboratory Systems 135 (2014) 110-125.

[25] R. Mathar, A. Žilinskas, On global optimization in two dimensional scaling, Acta Applicandae Mathematicae 33 (1993) 109-118. 
[26] I. Borg, P. Groenen, Modern Multidimensional Scaling: Theory and Applications, Springer, 1997.

[27] T. Cox, M. Cox, Multidimensional Scaling, Chapman and Hall, 2001.

[28] A. Buja, D. Swayne, Visualization methodology for multidimensional scaling, Journal of Classification 19 (2002) 743.

[29] A. Žilinskas, J. Žilinskas, Parallel hybrid algorithm for global optimization of problems occurring in MDS-Based visualization, Computers and Mathematics with Applications, 52(1-2) (2006) 211-224.

[30] D. Ko, R. Siriwardane, L. T. Biegler. Optimization of pressure swing adsorption and fractionated vacuum pressure swing adsorption processes for CO2 capture, Industrial \& engineering chemistry research, 44(21) (2005): 8084-8094. 